\title{
Tubeless percutaneous nephrolithotomy in renal allografts: a case report and mini-review
}

\author{
Siavash Falahatkar, Gholamreza Mokhtari, Samaneh Esmaeili, Seyed Morteza Bashiri Ebrahimian, \\ Nadia Rastjou Herfeh
}

Urology Research Center, Guilan University of Medical Sciences, Rasht, Iran

\begin{abstract}
Urinary lithiasis in transplanted kidney is a relatively uncommon complication. However, it may lead to a significant morbidity and loss of renal function. The report presents the case of a 32-year-old male renal-transplant recipient, with a stone in renal pelvis who was treated successfully by tubeless percutaneous nephrolithotomy (PCNL). The patient is currently stone free with no complication. This article also reviews in brief the treatment of this rare complication. Reviewing the literature showed, it could be noticed that PCNL is a safe and feasible procedure for treating nephrolithiasis in a transplanted kidney.
\end{abstract}

\section{Introduction}

The urological complications following renal transplantation have been reported in many studies. ${ }^{1-4}$ Among them urinary stone is a rare complication and its prevalence reported between $0.2 \%$ and $6.3 \% .^{1,2,5-8}$

Urinary calculi are often asymptomatic so it is difficult to diagnose them at an early stage by physicians ${ }^{1}$ and they are usually diagnosed when a sudden wors-

Correspondence: Samaneh Esmaeili, Urology Research Center, Razi Hospital, Rasht, Iran.

Tel./Fax: +98.1333525259.

E-mail: samaneh_815@yahoo.com

Key words: Lithiasis; calculi; tubeless; percutaneous nephrolithotomy; renal allografts; transplantation.

Acknowledgments: this study was supported by Urology Research Center, Guilan University of Medical Sciences.

Contributions: SF, study concept and design; SMBE, SE, acquisition of data; SF, SMBE, SE, NRH, drafting of the manuscript; SF, GM, SE, critical revision of the manuscript for important intellectual content; SF, GM, study supervision.

Conflict of interest: the authors declare no conflict of interest.

Received for publication: 10 July 2016

Accepted for publication: 30 August 2016.

This work is licensed under a Creative Commons Attribution NonCommercial 4.0 License (CC BY-NC 4.0).

CCopyright S. Falahatkar et al., 2017

Licensee PAGEPress, Italy

Italian Journal of Medicine 2017; 11:207-210

doi:10.4081/itjm.2016.759 ening kidney function happens or pyelonephritis is detected. ${ }^{2}$ The best and most accurate method to detect the size and location of the stone is ultrasound. ${ }^{3}$

Today, minimally invasive procedures are available to remove the graft stones. ${ }^{4}$ Percutaneous nephrolithotomy (PCNL) is an accepted procedure used for removing large kidney stone. ${ }^{9}$

Herein, we report on the case of a renal-transplant recipient with urinary stone that was successfully treated with PCNL, as well as a mini review of the relevant literature, is also presented.

\section{Case Report}

A 32-year-old male renal-transplant recipient, diagnosed with end-stage renal disease (ESRD) due to secondary hypertension, with fever and chills was admitted to Nephrology ward. The self-referred patient to hospital, suffered from increase in blood pressure. Urolithiasis in the transplanted kidney was diagnosed by ultrasonography (diagnosed accidentally without any specific complaint, any specific signs or symptoms). In the assessment, an increase in his creatinine was noticed and further studies confirmed diagnosis of ESRD. There was no evidence of a urinary tract infection/sepsis. The patient underwent dialysis three times a week.

An ultrasound scan in allograft kidney showed normal size, normal parenchyma and an $11 \times 19 \mathrm{~mm}$ renal stone in the renal pelvis.

In computed tomography scan report, both main patients' kidneys were atrophic with no stone and hydronephrosis. The parenchyma of transplanted kidney was preserved and a $25-\mathrm{mm}$ stone was found in the renal pelvis. Moderate to severe hydronephrosis was reported for this kidney (Figure 1). 


\section{Technique}

Surgical options were evaluated by the surgeon and PCNL was recognized as the best method of treatment.

Cystoscopy was performed for patient to find ureteral orifice for inserting ureter stent. At roof of the bladder, a bullous edema was detected but the orifice was not detected. After getting the calyceal access by ultrasound guidance, forthwith contrast material was injected to see the system clearly. For avoiding the possibility of losing the expected direction a guide wire was passed through Shiba needle into the transplanted kidney. Then the middle calyx access was performed fluoroscopically. By nephroscope two stones were visible in renal pelvic (one about $20 \mathrm{~mm}$ and the other about 7-8 mm). After lithotripsy, the stones were extracted. All accessible calyxes were evaluated by nephroscope. As there was no hematuria, double-J stent or nephrostomy was not placed and the surgery was totally tubeless. The stone was completely removed at the end of the procedure, which was confirmed by the ultrasonography 2 weeks later. The patient was discharged with full recovery from the hospital on the second day after the operation. Stone analysis revealed a mixed stone containing calcium oxalate (65\%) and calcium/phosphate (35\%).

\section{Discussion and Conclusions}

Allograft lithiasis is a rare but potentially devastating complication of kidney transplantation. ${ }^{9}$

Transplant recipients are more predisposed to developing urolithiasis due to their conditions such as: hyperfiltration, excessively alkaline urine, renal tubular acidosis, obstructive uropathy, recurrent urinary tract infections, and increased serum calcium caused by persistent tertiary hyperparathyroidism. ${ }^{10-14}$

Many series have reported the mean time of posttransplantation lithiasis between 1.6 to 3.6 years. ${ }^{4,15,16}$

As, this complication is usually asymptomatic, the diagnosis is often made incidentally by ultrasound examination followed by symptoms such as hematuria, oliguria/anuria, fever, elevated creatinine level, and positive urine culture. ${ }^{3,17}$

Traditionally, open surgery has been used for allograft calculus treatment which may lead to substantial morbidity and mortality., ${ }^{2} 18$

Nowadays, open surgery has increasingly been replaced by minimally invasive procedures such as extracorporeal shock wave lithotripsy (ESWL), flexible ureteroscopy and PCNL.,19

ESWL is the treatment of choice for un-obstructive renal calculi with diameter less than $1.5 \mathrm{~cm}^{1,20}$ but PCNL is the most effective procedure for those with large renal calculi. ${ }^{1,3,9,11,21}$

The extra-anatomic location of a renal allograft makes difficult the access to these kidneys and so overshadowed the decision to extract the stone. ${ }^{2}$ And also, the proximity to the bony pelvis causes that targeting the shock wave directly onto the stone becomes more difficult in ESWL. 1,4,9,10,22

But the anatomic position in the iliac fossa with the renal surface close to skin makes PCNL an attractive alternative and a feasible procedure in the treatment of urinary calculi. ${ }^{10}$

Many cases have been reported in which allograft lithiass was treated successfully with PCNL., ${ }^{2,9}$

PCNL has significant advantages in renal calculi extraction such as: need for minimal intra-operative bleeding, high stone-free rate, and renal access by one or maximum two attempts in tubeless approaches. , $^{93,24}$

In our case, the patient had a large stone in the renal pelvis. We performed tubeless PCNL for him
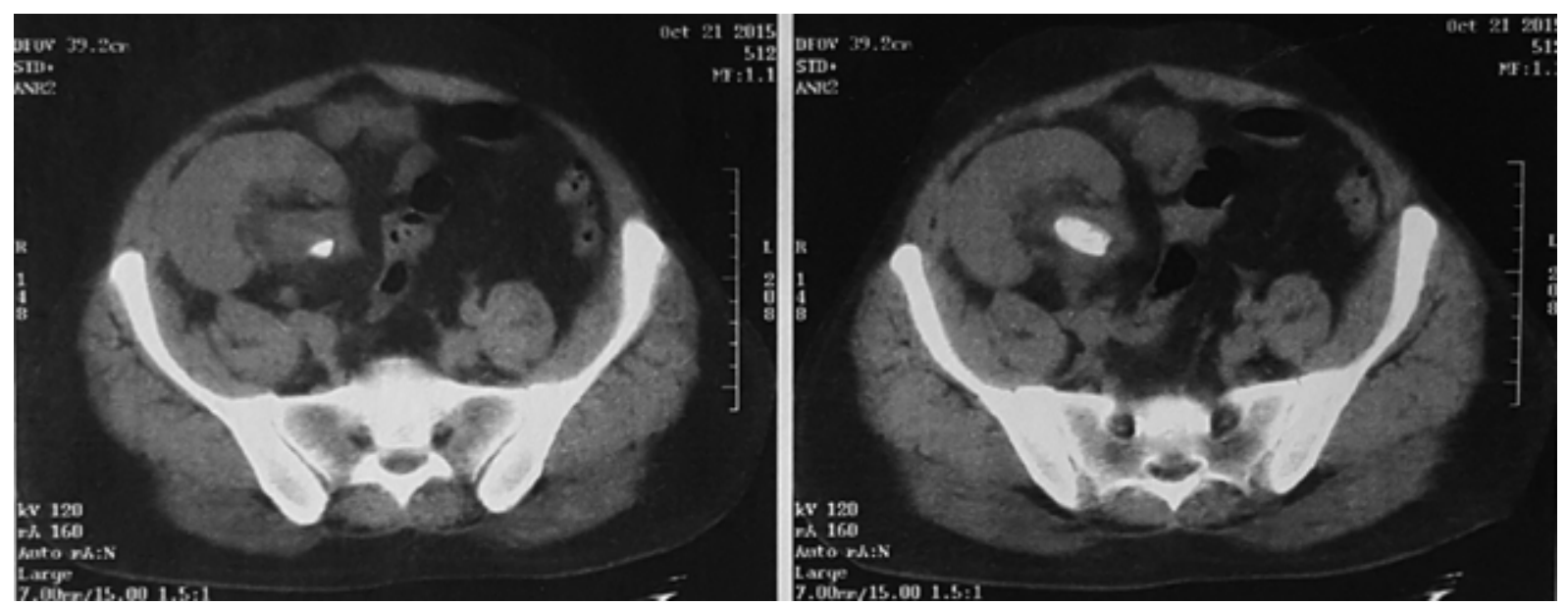

Figure 1. Axial (transverse) computed tomography image showing stone in the renal pelvis allograft. 
and removed the stone completely. By two months follow up, the patient was stone free without any complication.

Although, there are several reports about performing PCNL in pelvic and transplanted kidney which showed that the access to these kidneys is feasible by ultrasound and laparoscopic guided procedures, ${ }^{9-11}$ but we know PCNL in such kidneys heap upon the surgeon some technical and stress pressures.

Each intervention on transplanted kidney can bring a lot of emotional effect upon patients and their families, so it is mandatory for the surgeon to know each intricate details of procedure.

At first, we learned that insertion of ureteral catheter in transplanted kidney especially when kidney is affected by stone or other diseases is not so easy and perhaps it can be impossible, like in our case. If the surgeon is not able to insert the ureteral stent, it is better not to give up the procedure and continue it percutaneously.

Perhaps, sometimes attempting to insert a ureteral stent can be a waste of time. The transplanted kidney placed in pelvic fossa and the distance between skin and kidney was too much shorter than a normal kidney. ${ }^{10}$ The ultrasound was a useful guide to reach the kidney but discerning the exact point of calyx was a little difficult, because of that we preferred to inject contrast media to set all the structures of pyelocalyceal system.

We believe that the use of fluoroscopy for the access is more efficient than ultrasound and the surgeon is able to see the path apparently. On the other hand, injection contrast media helps the surgeon in finding again the calyx if it gets lost during the procedure.

In our case, the patient had a large stone in the renal pelvis. We performed tubeless PCNL for him and remove the stone completely. By two months follow up, the patient was stone free without any complication.

We found the middle calyx was a good way to reach the pelvic, although at first, we entered the lower calyx by ultrasound guided procedure. During the procedure, we perceived that the transplanted kidney was a little more fragile than normal kidney, so we recommend to all endourologists to be careful during the procedure and be more gently on it.

Although, there were many studies in the field of tubeless PCNL ${ }^{9,25}$ but tubeless PCNL in transplanted kidney needs more studies. Tubeless PCNL was found safe in this case.

Finally, PCNL in transplanted kidney is feasible and safe. The surgeon can perform tubeless PCNL case by case. Also, combine ultrasound and fluoroscopic guided PCNL in transplanted kidney makes the surgeon to enter the exact and better point into the calyx. Because of the fragility of transplanted kidney structures, the surgeon should be vigilant not to lacerate it.

\section{References}

1. Cicerello E, Merlo F, Mangano M, et al. Urolithiasis in renal transplantation: diagnosis and management. Arch Ital Urol Androl 2014;86:257-60.

2. Yuan HJ, Yang DD, Cui YS, et al. Minimally invasive treatment of renal transplant nephrolithiasis. World J Urol 2015;33:2079-85.

3. Mamarelis G, Vernadakis S, Moris D, et al. Lithiasis of the renal allograft, a rare urological complication following renal transplantation: a single-center experience of 2,045 renal transplantations. Transplant Proc 2014;46:3203-5.

4. Stravodimos KG, Adamis S, Tyritzis S, et al. Renal transplant lithiasis: analysis of our series and review of the literature. J Endourol 2012;26:38-44.

5. Crook TJ, Keoghane SR. Renal transplant lithiasis: rare but timeconsuming. BJU Int 2005;95:931-3.

6. Khositseth S, Gillingham KJ, Cook ME, Chavers BM. Urolithiasis after kidney transplantation in pediatric recipients: a single center report. Transplantation 2004;78:1319-23.

7. Lu HF, Shekarriz B, Stoller ML. Donor-gifted allograft urolithiasis: early percutaneous management. Urology 2002;59:25-7.

8. Zavos G, Pappas P, Karatzas T, et al. Urological complications: analysis and management of 1525 consecutive renal transplantations. Transplant Proc 2008;40: 1386-90.

9. McAlpine K, Leveridge MJ, Beiko D. Outpatient percutaneous nephrolithotomy in a renal transplant patient: World's first case. Can Urol Assoc J 2015;9:E324-8.

10. Wyatt J, Kolettis PN, Burns JR. Treatment outcomes for percutaneous nephrolithotomy in renal allografts. J Endourol 2009;23:1821-4.

11. Francesca F, Felipetto R, Mosca F, et al. Percutaneous nephrolithotomy of transplanted kidney. J Endourol 2002;16:225-7.

12. Harper JM, Samuell CT, Hallison PC, et al. Risk factors for calculus formation in patients with renal transplants. Br J Urol 1994;74:147-50.

13. Pei-Ling L, Gallery ED, Grigg R, et al. Renal function in unilateral nephrectomy subjects. J Urol 1992;147: $337-9$.

14. Chan JC. Physiological responses of the transplanted human kidney. J Urol 1973;110:162-5.

15. Kim H, Cheigh JS, Ham HW. Urinary stones following renal transplantation. Korean J Intern Med 2001;16: 118-22.

16. Challacombe B, Dasgupta P, Tiptaft R, et al. Multimodal management of urolithiasis in renal transplantation. BJU Int 2005;96:385-9.

17. Li SD, Wang QT, Chen WG. Treatment of urinary lithiasis following kidney transplantation with extracorporeal shock-wave lithotripsy. Chin Med J (Engl) 2011;124:1431-4.

18. Rhee BK, Bretan PN Jr, Stoller ML. Urolithiasis in renal and combined pancreas/renal transplant recipients. J Urol 1999;161:1458-62.

19. Hobart MG, Streem SB, Gill IS. Renal transplant complications. Minimally invasive management. Urol Clin North Am 2000;27:787-98. 
20. Montanari E, Zanetti G. Management of urolithiasis in renal transplantation. Arch Ital Urol 2009;81:175-81.

21. Krambeck AE, LeRoy AJ, Patterson DE, Gettman MT. Percutaneous nephrolithotomy success in the transplant kidney. J Urol 2008;180:2545-9.

22. Klinger HC, Kramer G, Lodde M, et al. Urolithiasis in allograft kidneys. Urology 2002;59:344-8.

23. Wang J, Zhao C, Zhang C, et al. Tubeless vs standard percutaneous nephrolithotomy: a meta-analysis. BJU Int 2011;109:918-24

24. de Cogain MR, Krambeck AE. Advances in tubeless percutaneous nephrolithotomy and patient selection: an update. Curr Urol Rep 2013;14:130-7.

25. Falahatkar S, Khosropanah I, Roshani A, et al. Tubeless percutaneous nephrolithotomy for staghorn stones. J Endourol 2008;22:1447-51. 